\title{
The Quiet Sun in Gamma Rays: Modeling Cosmic-Ray Electrons in the Inner Heliosphere
}

\author{
Elena Orlando* \\ Hansen Experimental Physics Laboratory and Kavli Institute for Particle Astrophysics and \\ Cosmology, Stanford University, Stanford, CA 94305, USA \\ E-mail: eorlandoestanford.edu
}

\section{Nicola Giglietto}

Dipartimento Interateneo di Fisica dell'Universita e Politecnico di Bari and INFN Sezione di Bari

\section{Igor Moskalenko}

Hansen Experimental Physics Laboratory and Kavli Institute for Particle Astrophysics and Cosmology, Stanford University, Stanford, CA 94305, USA

\section{Silvia Raino'}

Dipartimento Interateneo di Fisica dell'Universita e Politecnico di Bari and INFN Sezione di Bari

\section{Andrew Strong}

Max-Planck Institut für extraterrestrische Physik, Postfach 1312, D-85741 Garching, Germany

\begin{abstract}
The Sun in its quiescent state is a known gamma-ray source. The solar emission is produced by Cosmic Rays (CRs) penetrating the inner heliosphere and interacting with the solar atmosphere and optical photons. It is characterized by two spatially and spectrally distinct components: (i) the disk emission due to hadronic $\mathrm{CR}$ cascades in the solar atmosphere, and (ii) the spatially extended inverse Compton (IC) emission due to CR electrons scattering on the solar photons. The intensity of both components anti-correlates with the solar activity being the brightest during solar minima. Observations of the two components at various solar activities allow to gain information on CRs very close to the Sun and on CR propagation in the heliosphere. After the first observation of its gamma-ray emission in the EGRET archival data, Fermi-LAT is separating the two emission components with higher significance, allowing to precisely study the CR in the inner heliosphere. We present updates of the models of the IC emission based on recent CR measurements for various levels of solar activity, and we make predictions for e-ASTROGAM and AMEGO, proposed $\mathrm{MeV}-\mathrm{GeV}$ gamma-ray missions.
\end{abstract}

7th Fermi Symposium 2017

15-20 October 2017

Garmisch-Partenkirchen, Germany

${ }^{*}$ Speaker. 